\title{
Hair follicle units promote re-epithelialization in chronic cutaneous wounds: A clinical case series study
}

\author{
JIA-QI LIU $^{1 *}$, KONG-BO ZHAO ${ }^{2 *}, \mathrm{ZI}-H A O ~ F E N G^{1}$ and FA-ZHI QI ${ }^{1}$ \\ ${ }^{1}$ Department of Plastic Surgery, Zhongshan Hospital, Fudan University, Shanghai 200032; \\ ${ }^{2}$ Department of Surgery, Linyi People's Hospital, Linyi, Shandong 276000, P.R. China
}

Received June 9, 2014; Accepted February 5, 2015

DOI: $10.3892 / \mathrm{etm} .2015 .2465$

\begin{abstract}
Chronic cutaneous wounds are one of the most unfavorable pathophysiological processes in routine practice. However, developments in hair follicle unit therapy may aid the treatment of these wounds. The aim of the present study was to investigate the function of hair follicle units in chronic cutaneous wound re-epithelialization and to develop an effective protocol for wound treatment. A total of 14 patients, of which nine were male and five were female, with a mean age of 60.71 years (range, 19-76 years) and a mean wound area of $74.14 \mathrm{~cm}^{2}$, were treated in the study. The hair follicle units were dissected from a scalp graft and transplanted into the chronic cutaneous wound bed, after which clinical evaluation was performed. Images of the recipient site were captured at $0,1,2,3,4,5,8$ and 14 weeks following transplantation. In addition, histological examinations were conducted postoperatively at week 16 . Total wound re-epithelialization was observed in all the patients. Histological analysis revealed that the epidermis and papillary dermis were present in the wound area. Adnexal structures and the reticular dermis were also observed. Therefore, the present study demonstrated the ability of hair follicle units to promote chronic cutaneous wound healing.
\end{abstract}

\section{Introduction}

Previous decades have heralded the advancement of cell-based therapy and associated tissue regeneration and wound repair (1). The use of epithelial cells in tissue restoration and wound healing has attracted considerable interest. As such, the number of studies that seek to employ epithelial cells

Correspondence to: Dr Fa-Zhi Qi, Department of Plastic Surgery, Zhongshan Hospital, Fudan University, 180 Fenglin Road, Shanghai 200032, P.R. China

E-mail: fazhiqicn@163.com

\section{${ }^{*}$ Contributed equally}

Key words: cutaneous surgery, hair follicle, re-epithelialization, wound healing with high therapeutic potential to create novel therapies that can target and ultimately restore tissue integrity has been increasing (2-4). Chronic cutaneous wounds are one of the most unfavorable pathophysiological processes in dermatologic surgery; however, they may be treated using cell-based therapy development (5).

Cutaneous wound healing is a series of processes involving the epithelial, dermal and mesenchymal tissues, blood vessels, nerves and immune cells that act within a complex network of signals (6) to necessitate wound healing $(7,8)$. Re-establishing epithelial integrity is essential and the primary step to maintaining a regenerative response. The scalp has been identified as an excellent donor site for thin skin grafts (9); however, the scalp dermal graft presents a limited surface area and is $<3-4 \%$ of the total body surface area (10). Clinical and histological evidence has shown that the rapid healing of the scalp dermal graft is attributed to the differential potential of the cells in the hair follicles, and that re-epithelialization can also occur from grafted hair follicles (11). Numerous types of cells with multiple differentiation potentials have been identified in hair follicles, and their relative contribution to re-epithelialization has been recently determined (12). Considering the abundance of evidence suggesting the crucial role of hair follicles as re-epithelialization promoters, the notion of grafting hair follicle units harvested from the scalp into chronic cutaneous wounds was considered in the present study.

The primary aim of the present study was to develop an effective protocol for the treatment of chronic cutaneous wounds. The efficacy of the novel therapy that utilized hair follicle units to promote wound healing was investigated, in addition to the feasibility and potential healing capabilities of the hair follicle units that were transplanted into the chronic cutaneous wound bed. The transplanted hair follicle units were hypothesized to activate progenitor cells and promote re-epithelialization in the wound.

\section{Subjects and methods}

Patients. In this retrospective case series study, 14 patients with chronic cutaneous wounds, hospitalized at the Department of Plastic Surgery of Zhongshan Hospital (Shanghai, China) between June 2006 and September 2008, were recruited. All patients had previously received an autologous split-thickness skin graft; however, partial or completed necrosis had occurred 
and the graft was unable to heal independently. Eligible patients had chronic wounds that had persisted for $\geq 6$ weeks, were non-responsive to conventional treatment, with signs of delayed healing, and had a wound area of $\geq 5 \mathrm{~cm}^{2}$. Patients with alopecia, coagulopathy and other contraindications of the hair transplant technique, as well as elderly patients (>80 years), were excluded from the study. The study was conducted in accordance with the Declaration of Helsinki and with approval from the Ethics Committee of the Zhongshan Hospital of Fudan University. Written informed consent was obtained from all the participants.

Surgical procedures. Debridement of necrotic tissue was preoperatively performed on all the wounds. In all the patients, the donor area of the scalp was shaved. Following the application of adrenalized saline into the occipital area of the scalp, a $6.0 \times 1.5 \mathrm{~cm}$ scalp graft was harvested (Fig. 1). The hair follicle units were dissected from the graft (Fig. 2), and the units were subsequently transplanted onto the chronic cutaneous wound bed at a density of 4 units $/ \mathrm{cm}^{2}$, which was considered the minimum density required to guarantee tissue regeneration and re-epithelialization of the treated wound area. Care was taken to avoid hair follicle unit transection and bleeding was managed with pressure.

Following surgery, a Vaseline ${ }^{\circledR}$ gauze dressing (Shaoxing ZhengDe Surgical Dressing Co., Ltd., Zhejiang, China) was applied to the recipient site, which was changed daily until day 5, and every 3-5 days thereafter. The patients were not administered any painkillers or anticoagulants.

Assessment of clinical outcomes. Clinical evaluations were performed and digital images of the recipient sites were captured at weeks $0,1,2,3,4,5,8$ and 14 following intervention. An evaluation of the percentage of viable hair follicle units remaining at the wound site was conducted at week 2 . Clinical evaluation of wound healing was also performed. Wound healing was measured as the average reduction $(\%)$ in the total area of the wound bed at the intermediate time points, and was assessed by clinical and anatomopathological signs, including wound border retraction, the appearance of granulation tissue and evidence of re-epithelialization. To avoid misclassification bias, two independent plastic surgeons assessed the extent of re-epithelialization and measured the wound areas.

Histology. Histological analysis was performed postoperatively at week 16 , the end point of the study. A 4-mm punch biopsy was collected from the recipient wound and from the healthy skin around the wound for each patient using a biopsy trephine (\#w100671; Dongxiyi Technology Co., Ltd., Beijing, China). Samples were fixed in $10 \%$ formaldehyde and embedded in paraffin wax, using standard histological procedures. The samples were subsequently stained with hematoxylin and eosin. The samples were analyzed by a pathologist who was blinded to any clinical data to ensure the unbiased assessment of the secondary outcomes.

\section{Results}

Clinical outcomes. Table I shows the baseline clinical details of the patients. A total of 14 patients (male, 9; female, 5; mean age, $60.71 \pm 15.20$ years; median age, 61 years; age range, $19-76$ years)
Table I. Sample demographic and clinical characteristics of the patients $(n=14)$.

\begin{tabular}{lc}
\hline Characteristic & Result \\
\hline Age (years) & \\
Median (range) & $61(19-76)$ \\
Mean (SD) & $60.71(15.20)$ \\
Gender, n (\%) & \\
Male & $9(64.29)$ \\
Female & $5(35.71)$ \\
Reference wound area $\left(\mathrm{cm}^{2}\right)$ & \\
Median (range) & $48(26-220)$ \\
Mean (SD) & $74.14(58.51)$ \\
Reference wound duration (years) & \\
Median (range) & $2.5(0.5-13)$ \\
Mean (SD) & $4.18(3.39)$ \\
\hline
\end{tabular}

$\mathrm{SD}$, standard deviation.

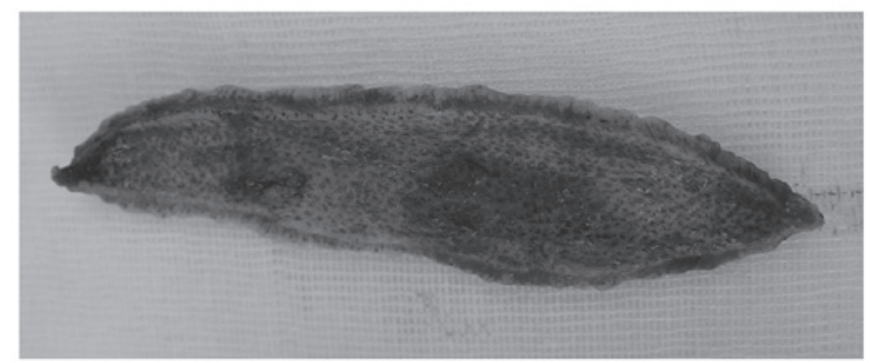

Figure 1. A strip of full-thickness scalp dermal graft. The long spindle shape limits secondary injury and is easily dissected.

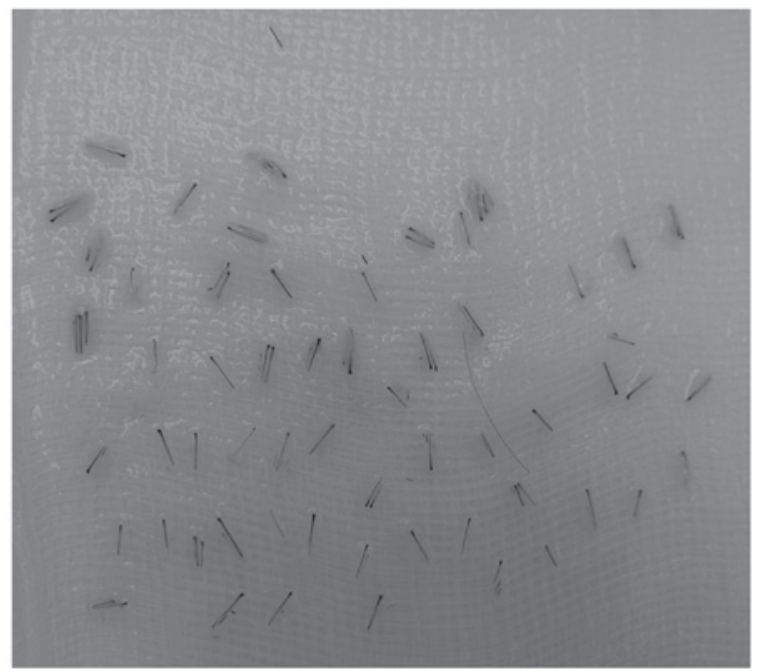

Figure 2. Hair follicles dissected from the scalp strip and isolated as units. The epidermis has been removed.

were treated using the novel protocol. The wound areas were relatively large, ranging between 26 and $220 \mathrm{~cm}^{2}$ (median area, $48 \mathrm{~cm}^{2}$; mean area, $74.14 \pm 58.51 \mathrm{~cm}^{2}$ ). A long wound duration (0.5-13 years) was also noted for the patients included in the 


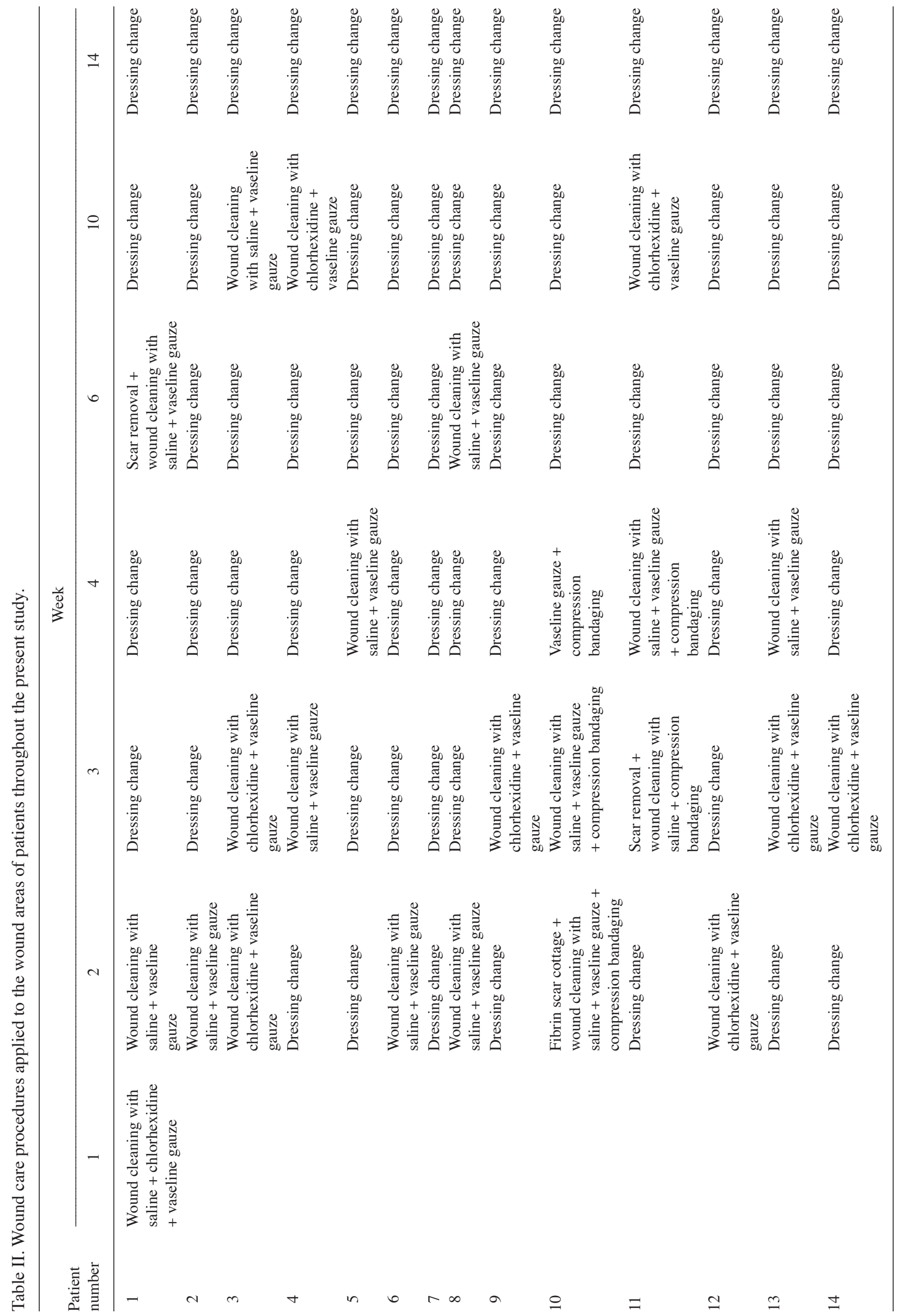




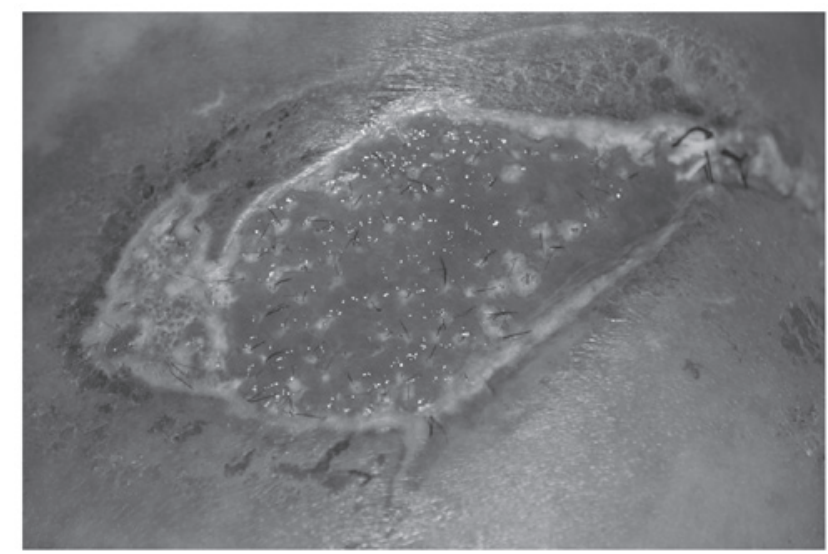

Figure 3. Patient 8 at week 2. Unviable hair follicle units faded away and a viability evaluation was able to be performed.

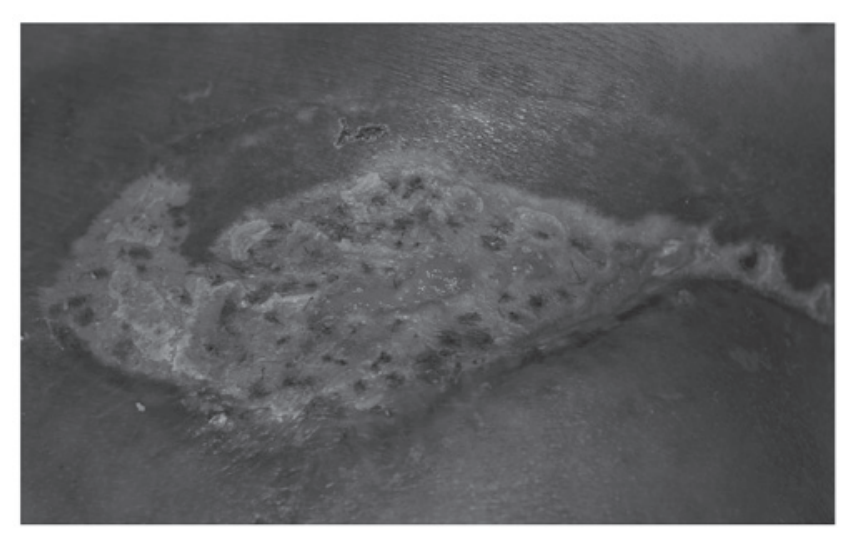

Figure 4. Patient 8 at week 5. Epithelialization around the hair follicle units can be observed. Thus, the wound reduction is significant.

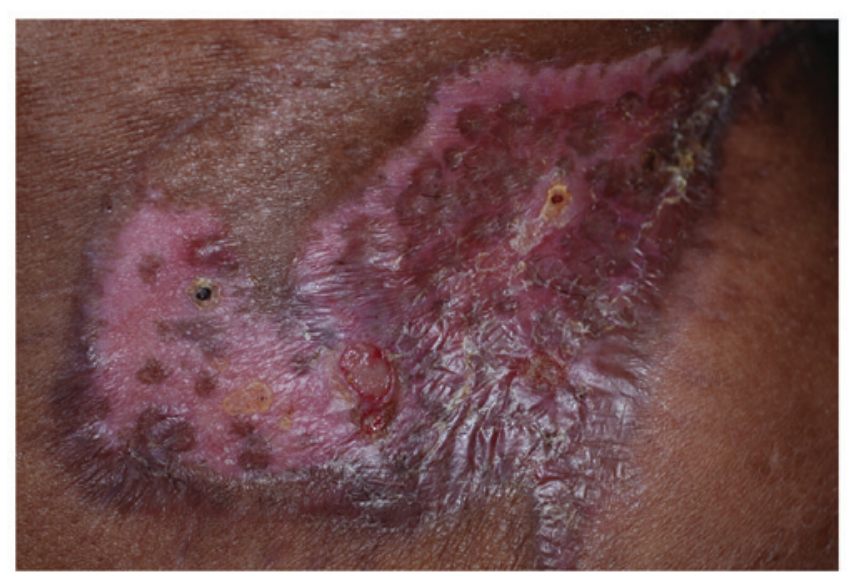

Figure 5. Patient 8 at week 8 . A reddish and fragile scar is observed.

study. Standard wound care procedures were applied in addition to the hair follicle unit therapy, and Table II reveals the details of the therapeutic regimens followed by each patient.

The average percentage reduction in the wound area was measured as the primary outcome. At week 1 , the wound bed was red and unviable hair follicle units were not observed. The viability evaluation at week 2 revealed the mean percentage of successful hair follicle units as $71.85 \pm 7.75 \%$ (Fig. 3). The fabric

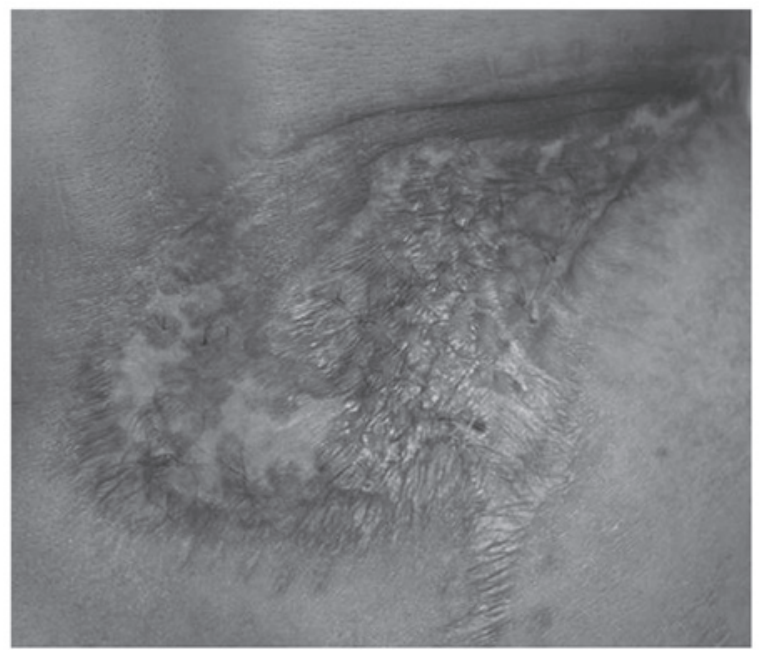

Figure 6. Patient 8 at week 14. Complete healing can be observed, which contributed to an improved healing quality.

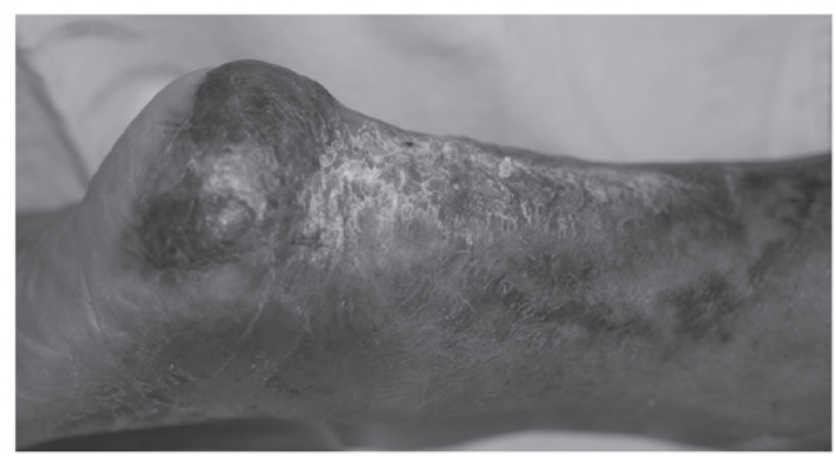

Figure 7. Patient 9 at week 14. Elasticity can be observed at the repair site.

scarred around the hair follicle units between weeks 3 and 4 . At week 5, clinical epithelialization was clearly observed and the wound reduction was significant (Fig. 4). Total healing was observed in all patients, and a fragile scar of reddish color was noted after the first 2 months (Fig. 5), which progressively disappeared (Fig. 6). Unlike the scar tissue, the reception area was elastic, which contributed to improved healing quality. Patient 9, who had a leg wound, developed enhanced ambulation as a result of the improved treatment method (Fig. 7). The disproportionate rarity of hair, in contrast to the viability of the hair follicle units, was also observed. Improvement in clinical symptoms, including wound border reactivation and a lower amount of exudation, was observed in 12/14 patients.

Complications. Overall, the procedure appeared to be safe. However, three patients presented with minor unrelated complications during the study period (patients 2 and 6 had atrial fibrillation, and patient $12 \mathrm{had}$ a recurrent edema in the lower right extremity).

Evaluation of the scalp-donor sites during the follow-up revealed no evidence of hair thinning, hair loss or alopecia. All donor sites were healed by day 7 following surgery.

Histological analysis. Punch biopsies were performed on the recipient square at week 16 following surgery. Histological 

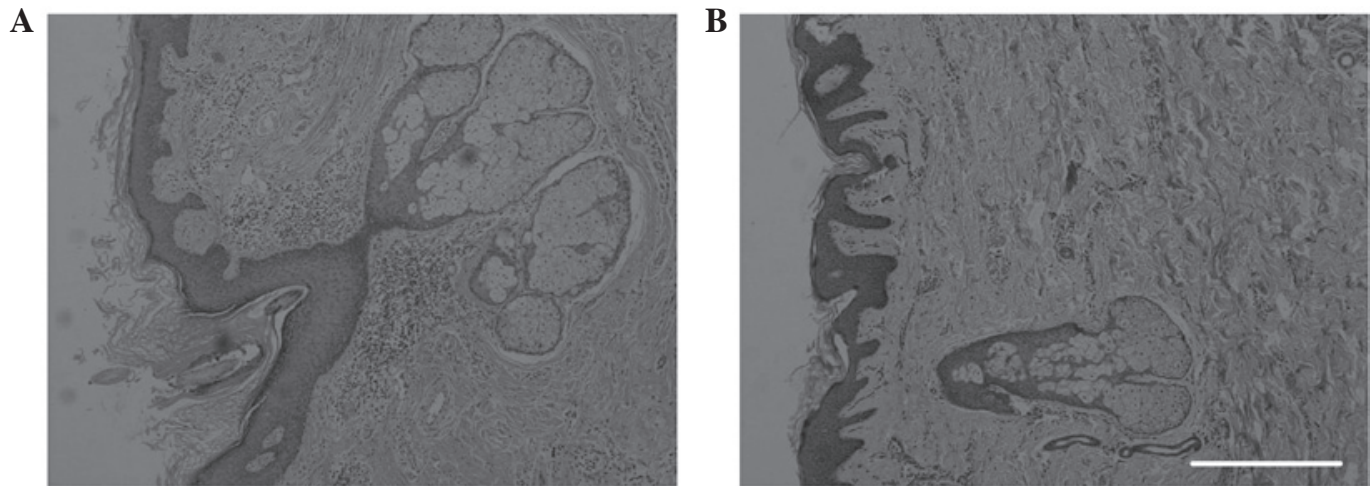

Figure 8. Histopathological examination evaluating the changes in the skin. (A) Skin from the restoration site and (B) healthy skin from around the wound (hematoxylin-eosin stain; original magnification, x10; scale bar, $200 \mu \mathrm{m}$ ).

analysis revealed that the epidermis and papillary dermis were contained within the recipient square. Adnexal structures and the reticular dermis were also observed. The outer hair shafts were macroscopically visible within the recipient wound area in the majority of patients (Fig. 8).

\section{Discussion}

Chronic cutaneous wounds, regardless of pathogenesis, can be devastating to patients, even when treated, and can result in a huge financial burden on medical establishments. Complete closure and protection of the wound is important for patients. Despite numerous therapeutic advances, the gold-standard protocol used in routine practice is an autologous split-thickness skin graft. This procedure involves removing a piece of skin from a secondary surgical site and reapplying the graft onto the wound bed. Although the clinical outcome of the skin graft treatment is reasonable, donor sites are limited and secondary injury is inevitable. Technological developments, including suspending cells in fibrin-collagen gel (13), have been investigated in an attempt to obtain an improved treatment for wound healing. However, these are costly to produce and are not covered by Chinese health insurance companies.

The epithelium can be restored by the migration of epithelial cells from the old epithelium adjoining a wound or by centrifugal migration from any hair follicle that remains within the wound (14). Several studies have unambiguously identified the hair follicle as a major reserve of adult stem cells $(15,16)$. Dermal grafts from the scalp (10), early implantation of microdissected hair follicles through a silicone epidermis (11) and hair grafting (17) are used for cutaneous wound repair; however, the procedures are complicated. In the present study, hair follicles without epidermal or interfollicular tissue were transplanted. The hair follicles were microdissected as individual units and transplanted into the wound bed. The function of the hair follicles as promoters of re-epithelialization was observed in the current study.

The present study was originally conceived as research into the feasibility of a novel therapy. Questions relating to the surgical technique were not specifically assessed; however, placing the hair follicles onto the wound (partially trialled on patients 1 and 2) resulted in a lower degree of viability compared with other patients in the present study (data not shown). Patient 1 displayed a relatively large wound area $\left(186 \mathrm{~cm}^{2}\right)$ and patient 2 had a long wound duration (13 years, including primary and repeating recurrent).

The clinical results indicate that the method of transplanting hair follicle units into the cutaneous wound is not only feasible in practice, but is also successful in improving the long-term clinical healing of wounds. In all the cases, the hair follicles augmented epithelialization and the formation of granulation tissue at the wound site. At the study endpoint, the hair follicle-treated wound area appeared to be elastic, unlike scar tissue, which contributed to the improved healing quality and allowed enhanced ambulation for one patient with a wounded leg. Although complete healing was observed in all the patients, an objective healing rate should be based on more substantial data and stronger evidence. A larger sample size is required in future studies.

Histological analysis provided evidence of re-epithelialization and the existence of outer hair shafts. The recovered area was likely to have contained a number of adnexa of the skin. However, when analyzing the histological results, the exact position of the biopsies may differ due to the varying overall aspect of the entire wound.

Recent advances in epithelial stem cell biology have improved the understanding of the role of hair follicles in wound healing mechanisms, and have provided a basis for the development of novel therapies (18). Various follicular cells contribute to wound healing through different methods. Bulge stem cells produce transiently amplifying epidermal progeny, while non-hair follicle-fated isthmus stem cells generate long-lasting populations in the wound epidermis $(19,20)$. Clinical and experimental data indicate that the involvement of follicle-derived dermal cells results in qualitatively improved dermal repair $(18,21)$. Consistent with previous studies, the observations of the present study support the hypothesis that hair follicles perform an important function in the repair of cutaneous wounds $(22,23)$. Prior to the dissection of hair follicle units, the epithelium was intentionally removed from the graft. This step allowed for the formal exclusion of the possibility that the healing effect was due to non-follicular tissue. However, in the current study, the limited sample size (which was small since the study was the first to be conducted in clinical practice) and experimental methods may have affected the quality of the results; the data are unable to confirm that re-epithelialization occurred from the stem cells specifically. Nevertheless, wound healing initiated by the 
follicles applied to the chronic wound bed was observed and may have indirectly supported the hypothesis that hair follicles perform an important function in the repair of cutaneous wounds. Although there have been numerous advances in stem cell therapy, further studies are essential.

In conclusion, the present study demonstrated the capacity of hair follicle units to repair chronic cutaneous wound sites. Complete healing was observed and the procedure appeared to be safe. With the exclusion of alopecic, insufficiently hairy and elderly patients, the present method may be used when donor sites are rare, due to the reduced donor-site morbidity, and may be a novel surgical method to avoid more complicated, costly and time-consuming procedures. By combining the hair transplantation and plastic reconstruction techniques, a novel surgical protocol may be developed, which may provide an additional option for secondary surgery on chronic wounds. However, this method requires a surgeon with advanced training in hair follicle unit harvesting.

\section{Acknowledgements}

The authors thank the patients who participated in the study. The study was supported by a grant provided by the Shanghai Municipal Science and Technology Commission (\#07JC14013; 2007).

\section{References}

1. Castilla DM, Liu ZJ, Tian R, Li Y, Livingstone AS and Velazquez OC: A novel autologous cell-based therapy to promote diabetic wound healing. Ann Surg 256: 560-572, 2012.

2. Lau K, Paus R, Tiede S, Day P and Bayat A: Exploring the role of stem cells in cutaneous wound healing. Exp Dermatol 18 921-933, 2009.

3. Hu L, Zhao J, Liu J, Gong N and Chen L: Effects of adipose stem cell-conditioned medium on the migration of vascular endothelial cells, fibroblasts and keratinocytes. Exp Ther Med 5: 701-706, 2013.

4. Dittmer J and Leyh B: Paracrine effects of stem cells in wound healing and cancer progression (Review). Int J Oncol 44: 1789-1798, 2014

5. Brower J, Blumberg S, Carroll E, Pastar I, Brem H and Chen W: Mesenchymal stem cell therapy and delivery systems in nonhealing wounds. Adv Skin Wound Care 24: 524-534, 2011.

6. Jahoda CA and Christiano AM: Niche crosstalk: Intercellular signals at the hair follicle. Cell 146: 678-681, 2011.
7. Murawala P, Tanaka EM and Currie JD: Regeneration: The ultimate example of wound healing. Semin Cell Dev Biol 23: 954-962, 2012.

8. Waters JM, Richardson GD and Jahoda CA: Hair follicle stem cells. Semin Cell Dev Biol 18: 245-254, 2007.

9. Mimoun M, Chaouat M, Picovski D, Serroussi D and Smarrito S: The scalp is an advantageous donor site for thin-skin grafts: A report on 945 harvested samples. Plast Reconstr Surg 118: 369-373, 2006

10. Zakine G, Mimoun M, Pham J and Chaouat M: Reepithelialization from stem cells of hair follicles of dermal graft of the scalp in acute treatment of third-degree burns: first clinical and histologic study. Plast Reconstr Surg 130: 42e-50e, 2012.

11. Navsaria HA, Ojeh NO, Moiemen N, Griffiths MA and Frame JD: Reepithelialization of a full-thickness burn from stem cells of hair follicles micrografted into a tissue-engineered dermal template (Integra). Plast Reconstr Surg 113: 978-981, 2004.

12. Plikus MV, Gay DL, Treffeisen E, Wang A, Supapannachart RJ and Cotsarelis G: Epithelial stem cells and implications for wound repair. Semin Cell Dev Biol 23: 946-953, 2012.

13. Skardal A, Mack D, Kapetanovic E, Atala A, Jackson JD, Yoo J and Soker S: Bioprinted amniotic fluid-derived stem cells accelerate healing of large skin wounds. Stem Cells Transl Med 1: 792-802, 2012.

14. Brown JB and McDowell F: Epithelial healing and the transplantation of skin. Ann Surg 115: 1166-1181, 1942.

15. Jaks V, Kasper M and Toftgård R: The hair follicle - a stem cell zoo. Exp Cell Res 316: 1422-1428, 2010.

16. Jiménez F, Garde C, Poblet E, et al: A pilot clinical study of hair grafting in chronic leg ulcers. Wound Repair Regen 20: 806-814, 2012.

17. Jahoda CA, Horne KA and Oliver RF: Induction of hair growth by implantation of cultured dermal papilla cells. Nature 311: 560-562, 1984

18. Hill RP, Gardner A, Crawford HC, et al: Human hair follicle dermal sheath and papilla cells support keratinocyte growth in monolayer coculture. Exp Dermatol 22: 236-238, 2013.

19. Jensen KB, Collins CA, Nascimento E, Tan DW, Frye M, Itami S and Watt FM: Lrig1 expression defines a distinct multipotent stem cell population in mammalian epidermis. Cell Stem Cell 4: 427-439, 2009

20. Snippert HJ, Haegebarth A, Kasper M, et al: Lgr6 marks stem cells in the hair follicle that generate all cell lineages of the skin. Science 327: 1385-1389, 2010.

21. Jahoda CA and Reynolds AJ: Hair follicle dermal sheath cells: unsung participants in wound healing. Lancet 358: 1445-1448, 2001.

22. Leirós GJ, Kusinsky AG, Drago H, et al: Dermal papilla cells improve the wound healing process and generate hair bud-like structures in grafted skin substitutes using hair follicle stem cells. Stem Cells Transl Med 3: 1209-1219, 2014.

23. Baquerizo Nole KL and Kirsner RS: Hair follicles and their potential in wound healing. Exp Dermatol 24: 95-96, 2015. 